\title{
Assistência à saúde dos idosos vítimas de acidentes e violência: uma análise da rede de serviços SUS no Recife (PE, Brasil)
}

\author{
Health care for aged victims of accidents and violence: \\ analysis of SUS health services in Recife (PE, Brazil)
}

\author{
M aria Luiza Carvalho de Lima ${ }^{1}$ \\ Edinilsa Ramos deSouza ${ }^{2}$ \\ M aria Luiza LopesTimóteo de Lima ${ }^{1}$ \\ AliceKelly Barreira ${ }^{1}$ \\ Eduardo Duque Bezerra ${ }^{1}$ \\ Raquel Moura LinsAcioli ${ }^{1}$
}

\footnotetext{
${ }^{1}$ Laboratório de Estudos em ViolênciaeSaúdedo Departamento deEstudos em Saúde Coletiva, Centro de Pesquisas Aggeu M agalhães, Fundação Oswaldo Cruz/PE. Campus da UniversidadeFederal de Pernambuco. Av. Moraes Rego s/n. 50670-420 Recife PE.

luiza@cpqam.fiocruz.br ${ }^{2}$ Centro Latino-Americano deEstudos de Violência e Saúde Jorge Carelli, Escola Nacional deSaúdePública Sergio Arouca, Fundação Oswaldo Cruz.
}

Abstract A situational diagnosis of the health services regarding the care of aged victims of accidents and violence (AVAV) was carried out in Recife, Pernambuco, Brazil. The National Policy for Reducing Accident and Violence Related M orbidity and $\mathrm{M}$ ortality and the National Policy for the Aged People $\mathrm{H}$ ealth were used as references. The methodology was based on thetriangulation method, with both quantitative and qualitative approaches. Questionnaires and interviews were answered by managers and health staff of hospital, prehospital and rehabilitation services; and local aged health policy managers. In 2006, only the Family H ealth Program reported prehospital care for AVAV, 31 cases were due to violence and 18 to accidents. The hospital care for aged people was $7,2 \%$ of the total care, $27 \%$ from accidents and $10 \%$ from violence. In the sameyear, there was no record of rehabilitation care of AVAV. The directives of the policies studied are only partially followed. The health care is deficient in several aspects, such as: clinical protocols; notification devices; support to the aged, caregivers and aggressors; and also continuous training. This analysis can be such a contribution to the reorganization of the local health system, recognizing the aged person as vulnerable to accidents and violence. Key words Aged, Violence, Accidents, $\mathrm{H}$ ealth services, $\mathrm{H}$ ealth policy
Resumo Foi realizada análise diagnóstica dos serviços de saúde com relação à atenção aos idosos vítimas de acidentes e violência (IVAV) no Recife (Pernambuco, Brasil) com base nas diretrizes da Política Nacional de Redução da M orbimortalidadepor Acidentes eV iolência eda Política N acional de Saúde da Pessoa Idosa. A investigação se pautou na triangulação de métodos, com abordagens quantitativa e qualitativa. Gestores e profissionais de serviços deatenção pré hospitalar, hospitalar, reabilitação eresponsáveis pela saúdedo idoso no município responderam a questionárioseentre vistas. Em 2006, 0 atendimento pré hospitalar para IVAV foi relatado apenas pelo Programa de Saúde da Família, sendo 31 casos por violências e 18 por acidentes; para o hospitalar, 7,2\% corresponderam ao atendimento de idosos, e desses, 27\% acidentes e $10 \%$ violência; em reabilitação não houve registro de atendimento a IVAV. As diretrizes das políticas estudadas são contempladas apenas parcialmente e 0 atendimento é deficiente em relação a diversos aspectos, como protocolos clínicos; notificação; suporte aos idosos, cuidadores e vitimizadores; ecapacitação profissional. Essa análise pode representar uma contribuição na reordenação do sistema local de saúde, reconhecendo a pessoa idosa como vulnerável a acidentes e violência. Palavras-chave Idoso, Violência, Acidentes, Serviços de saúde, Política de saúde 
Introdução

A violência contra a pessoa idosa ainda transita por um campo deinvisibilidades, apesar de muito frequentena dinâmica familiar da maioria das cidades do Brasil. 0 tema ainda é pouco investigado empiricamente, e esseé um fenômeno social que merece uma atenção especial pelas repercussões na saúde de um grupo populacional vulnerável. Contudo, a constituição de políticas públicas vem configurando estratégias de enfrentamento dessefenômeno, cuja raiz social e estreita relação familiar associam-se ao mito da velhice inútil para se cobrir de silêncio.

A cidade do Recife, detentora de um perfil diferenciado de envel hecimento, ocupa a 3 a posição em número de idosos entre as cidades brasileiras, com $9,4 \%$, portanto tem interesse nessa discussão $0^{1}$. De acordo com a Organização M undial da Saúde (OM S), uma comunidade com 7\% de pessoas idosas pode ser considerada uma população envelhecida ${ }^{2}$, ainda que ela ocupe a 19a posição com relação à expectativa de vida ${ }^{2}$. Certamente, o fato de a cidade do Recife estar entre as mais violentas do país influencia esse perfil.

A pesar dos indicadores da capital pernambucana, ela é parte de uma dinâmica de envelhe cimento que acontece praticamente da mesma forma em cidades de semel hante porte e importância regional. 0 rápido aumento da esperança de vida, o crescimento na participação social da pessoa idosa ea incorporação de políticas públicas voltadas à questão etária são reflexos da transição demográfica em curso no Brasil.

Diferente das doenças transmissíveis, o entorno do fenômeno violento se mostra bem mais complexo. Dentre tantos fatores envolvidos em seus modelos explicativos, a redução da mobilidade física pode ser tomada como exemplo para se compreender, ainda que em parte, a violência contra os velhos e velhas. Estes se tornam mais suscetíveis ao que D ebert ${ }^{3}$ classifica como segregação espacial do idoso. Outros fatores podem contribuir para o aumento da ocorrência de lesões por causas externas na população idosa, tais como problemas visuais, neurológicos, declínio da função mental e uso de substâncias psicoativas $^{4}$ e doenças associadas 5 .

Esseéum tipo deocorrência preferencialmente doméstica, com baixo potencial de denúncia e cujo agressor é familiar ou pessoa próxima ${ }^{6}$. As violências se manifestam de diferentes maneiras: incluem violência física, psicológica, financeira, sexual, o abandono, a negligência e a autonegligência?.
Em 2006, a cidade do Recife contabilizou 201.474 internações hospitalares por todas as causas, com uma média de 7,1/1.000 habitantes. Do total de internações, 9,1\% foram por causas externas, sendo o quarto lugar. A taxa de internação por causas externas variou de 3,9/1.000 habitantes em 2000 para 6,4 em 2006, o que representa um aumento de $64,6 \%{ }^{8}$.

Entre os anos de 2000 e 2006, o maior crescimento no número de internações foi verificado para as neoplasias e as lesões (envenenamentose outras consequências de causas externas), com $137,7 \%$ e $62,4 \%$, respectivamente. N esse período, ocorreu um crescimento de $57,9 \%$ no total de internações por causas externas em idosos, com particularidades nas causas específicas: 0 número de internações por acidentes de transporte triplicaram, enquanto as internações por outras causas externas se reduziram. Conforme o aumento da idade, diminuem as internações por acidentes de transporte e aumentam os internamentos por outras causas externas de lesões acidentais $s^{8}$.

A carência na operacionalização deuma política de saúde em conformidade com os princípiose diretrizes do Sistema Ú nico de Saúde(SU S) é reproduzida em maior ou menor grau em todo o território nacional. Essa desigualdade, por assim dizer, injusta, podeser definida como iniquidade e atinge os idosos mais fortemente.

A cidadedo Recifenão foge a essa real idade, $\mathrm{e}$ sua rede assistencial de saúde deve se estruturar para o enfrentamento da violência contra a pessoa idosa. A média de permanência desse público em serviços especializadosé maior quando comparada a outros grupos etários. 0 público de 70 a 79 anos, em 2006, experimentou 115,8 dias de internação por essa natureza de transtorno, enquanto a população de 30 a 39 anos permaneceu, em média, 66,5 dias 8 .

Especificamente, a Política Nacional de Redução da M orbimortalidade por Acidentes e Violência (PN RM AV) ${ }^{9}$ ea Política Nacional deSaúde da Pessoa Idosa ${ }^{10}$ contemplam em boa parte das suas diretrizes a sistematização, organização, reestruturação, consolidação e ampliação da assistência à saúde.

A presente pesquisa teve como objetivo realizar uma análise diagnóstica dos sistemas de saúde com relação à atenção prestada aos idosos vítimas de acidentes e violências no Recife, com base nas orientações das princi pais diretrizes das políticas dirigidas a essegrupo, dentre as quais se encontram a PNRM AV e a Política Nacional de Saúde da Pessoa Idosa. 


\section{Método}

Os resultados apresentados fazem parte de um estudo maior realizado em cinco capitais do país: Recife, Rio de Janeiro, Brasília, Curitiba e Manaus, porém, 0 artigo apresentará apenas as informações referentes à cidade do Recife. Os parâmetros norteadores para a análise das ações desaúdeforam definidostomando-se como base as principais políticas de atendimento integral ao idoso: a PN RM AV, a Política Nacional de Saúde da Pessoa Idosa, a Política Nacional de Saúde M ental, o Estatuto do Idoso ea Política Nacional de Saúde.

A pesquisa se constituiu em um trabalho de equipeinterinstitucional einterdisciplinar e teve sua coordenação geral por pesquisadores do Centro Latino-Americano deEstudos deViolência e Saúde Jorge Careli (Claves), Escola N acional de Saúde Pública Sergio Arouca (Ensp), Fundação Oswaldo Cruz (Fiocruz/RJ) e coordenação local no Recife, por pesquisadores do Centro de Pesquisa Aggeu M agalhães (Fiocruz/PE).

A investigação se pautou nos princípios da triangulação de método ${ }^{11,12}$, integrando as abordagens quantitativa e qualitativa. Todos os instrumentos foram construídos com o intuito de investigar o cumprimento das diretrizes e parâmetros estabelecidos nas políticas que norteiam 0 atendimento à pessoa idosa vítima de acidente eviolência.

A abordagem quantitativa contou com cinco instrumentos de coleta de dados. Um questionário foi aplicado na fase inicial de mapeamento dos serviços que atendem idosos vítimas de acidentes eviolência. As questões foram distribuídas em dois blocos: um relativo à identificação da unidade de saúde e o segundo visando caracterizar as atividades de atendimento aos idosos em situação deacidentes/violências. Outros trêsquestionários destinados aos serviços clínicos (pré hospitalar, hospitalar, reabilitação) foram elaborados e aplicados na segunda fase, visando qualificar 0 atendimento e as ações oferecidas. Todos estiveram compostos por quatro blocos de questões referentes à identificação, à estrutura e à organização do serviço e ao registro dos dados.

$\mathrm{Na}$ abordagem qualitativa foram feitas, na primeira fase, entrevistas com gestores responsáveis pela atenção pré-hospitalar, hospitalar e de reabilitação, além dos responsáveis pela área de saúde do idoso do município. Na segunda fase, foram elaborados oito roteiros de entrevistas que envolveram os seguintes gestores e profissionais: coordenador/diretor, médico e socorrista, coor- denador do serviço de reabilitação efisioterapeuta, coordenador de centro de referência no atendimento ao idoso e gerontólogo, gestor responsável pela administração dos hospitais de emergência e médico ortopedista ou urgentista.

Os bancos de dados quantitativos foram convertidos para o softwareSPSS versão 13.0, no qual serealizou a categorização das questões abertas e frequência simples e relativa. Os dados qualitativos foram analisados seguindo os princípios da Análise deConteúdo, modalidadetemática adaptada da proposta de Bardin ${ }^{13}$. A análise de conteúdo foi conduzida basicamentepor um recorte interpretativo ${ }^{14}$.

Todos os procedimentos éticos exigidos pela Resolução no 196/96 do Conselho Nacional de Saúde, que regulamenta pesquisas com seres humanos, foram seguidos. 0 projeto foi aprovado pelo Comitê de Ética em Pesquisa da Ensp/Fiocruz e das Secretarias de Saúde dos municípios, às quais também foi solicitada permissão para a realização do estudo nas instituições. Todos os sujeitos envolvidos foram devidamente esclarecidos quanto às implicações advindas de sua participação no trabalho e assinaram o termo de consentimento para a aplicação dos questionários e das entrevistas.

\section{Resultados ediscussão}

A PNRM AV ${ }^{9}$ possui sete diretrizes, das quais quatro fazem referência à assistência à saúde: sistematização, ampliação e consolidação do atendimento pré-hospitalar; assistência interdisciplinar e intersetorial às vítimas de acidentes e de violências; estruturação e consolidação do atendimento voltado à recuperação e à reabilitação; capacitação de recursos humanos. Na Política Nacional de Saúde da Pessoa Idosa ${ }^{10}$, das nove diretrizes, quatro fazem tal referência: atenção integral eintegrada à saúde da pessoa idosa; estímulo às ações intersetoriais; formação e educação permanente dos profissionais de saúde do SUS na área de saúde da pessoa idosa; divulgação e informação sobre a Política Nacional de Saúde da Pessoa Idosa para profissionais de saúde, gestores e usuários do SUS.

Sistematização, ampliação e consolidação do atendimento pré-hospitalar

0 atendimento pré-hospitalar compreendea esfera de atenção às urgências que possui componentes pré hospitalares fixos: unidades bási- 
cas de saúde e unidades de saúde da família (USF), equipes de agentes comunitários de saúde, ambulatórios especializados, serviços de diagnóstico eterapias, e unidades não hospitalares de atendimento às urgências; além de componentes pré-hospitalares móveis: Serviço de Atendimento M óvel deUrgência (Samu) eos serviços associados de salvamento e resgate.

A diretriz da PN RM AV ${ }^{9}$ que preconiza a "sistematização, ampliação e consolidação do atendimento pré-hospitalar" contempla principalmente o pré-hospitalar móvel. No entanto, no presenteestudo, foram investigadas dezoito unidades da atenção pré-hospitalar fixa e móvel. Dessas, seis são USF, as de maior demanda em cada distrito sanitário; quatro serviços de PS 24h no município do Recife, além do Samu. Os sete restantes são ambulatórios especializados, sendo cinco serviços de assistência ao idoso e dois ambulatórios especializados em atendimento de mulheres vítimas de violência.

$\mathrm{Na}$ cidade do Recife, a demanda espontânea nos serviços de urgência é muito grande. Além da falta de estrutura e pessoal para atender adequadamenteao fluxo de pacientes, existeum grande número de casos que poderiam ser atendidos no setor ambulatorial, como pode ser registrado na fala do profissional de saúde: 0 fluxo égrande em virtude do ambulatório não funcionar adequadamente. Faltam profissionais, a marcação é difićlima e, então, pessoas com lombalgias, dores crônicas, procuram a urgência. Esse é o grande problema das urgências, porque sobrecarrega o setor, e a qualidade do atendimento termina caindo muito por causa da grande quantidade de pacientes, poucos profissionais e más condições de trabalho (médico ortopedista de SPA).

Do total de 711.852 atendimentos préhospitalares realizados e registrados em 2006 nas unidades analisadas, o maior percentual deatendimento ao idoso ocorreu nas USF, perfazendo um total de $27,5 \%$, o queécondizentecom a proximidade dessas unidades das residências e com a facilidade de acesso dos idosos. Com relação ao atendimento direcionado para idosos vítimas de acidentes e violências, as unidades do Programa de Saúde da Família (PSF) também se destacam por serem as únicas a relatarem este tipo de atendimento, sendo 31 por violências e 18 por acidentes.

Apesar de existirem ambulatórios especializados para o atendimento do idoso, não houve registro de casos de violências nestas unidades em 2006, o que permite interrogar até que ponto os geriatras e gerontólogos estão sensibilizados para a questão da violência no idoso. Também chama a atenção o fato de os serviços de PS $24 \mathrm{~h}$ terem registrado o maior número de atendimentos (34.026) e, no entanto, nenhum registro de atendimento de idosos.

Essa não é uma realidade estritamente brasileira. De acordo com estudo realizado em Ohio, EUA, ao avaliar a experiência, conhecimentos e atitudes de 250 médicos defamília e 250 internistas gerais, foi observado que os médicos da família eram os mais sensibilizados, porém mais de $60 \%$ dos clínicos disseram nunca ter perguntado ou investigado nos seus pacientes suspeita demaus-tratos contra o idoso ${ }^{15}$. Esses dados alertam para duas questões: a necessidade de profissionais capacitados para uma ausculta diferenciada e sensível quanto à abordagem da violência na terceira idadee a ausência de ficha de notificação e de um fluxo bem definido nos serviços de saúde - questões essas reforçadas pelo discurso de gestores, unânimes em afirmar a não existência do atendimento específico ao idoso vítima de acidentes eviolência no município.

Quanto aos itens necessários relativos à estrutura das unidades, observou-se certa adequação ao selevar em conta o perfil de cada um deles. Entretanto, destacou-seo fato de apenasumaUSF possuir cadeira de rodas e três PS 24h não possuírem acesso diferenciado para ambulância.

Foi identificada a presença de profissionais segundo sua especialidade compondo o mínimo adequado, de acordo com o perfil dessas unidades. $\mathrm{E}$ todos os ambulatórios especializados em assistência aos idosos contam com médico geriatra, o que corresponde à exigência do Estatuto da Pessoa Idosa ${ }^{16}$ que pontua a necessidade de atendimento geriátrico e gerontológico em ambulatórios.

A investigação solicitou aos profissionais/gerentes uma avaliação (nota) do atendimento realizado pelas unidades de saúde. 0 Samu atribuiu a maior nota ao transporte de pacientes, enquanto os demais serviços destacaram a variedade de oferta de medicamentos.

As menores notas foram atribuídas aos seguintes itens: fluxos de referência e contrarreferência, pelasUSF; capacidade da equipepara atender idosos em situação deviolência, pelosPS24h; transporte de pacientes, pelos ambulatórios especializados; edisponibilidade devagas/leitos em outros serviços, pelo Samu.

Com relação à dificuldade dese obter um leito hospitalar, o problema é generalizado pela superlotação dos hospitais, portanto esta situação não é exclusiva dos idosos. Os gestores mencionaram, entretanto, que lidar com um paciente idoso traz 
mais dificuldades etorna-seainda mais complexo quando há a necessidade de transferência para outros hospitais e não há uma vaga imediata. 0 idoso tem muita comorbidade e, frequentemente, 0 fato dele ser hipertenso, diabético, cardiopata, exige UTI. M uitas vezes um paciente idoso é transferido para um hospital referência quenão dispõe deUTI, então o pacente volta. I sso aí éa grande dificuldade do idoso para conseguir vagas de leitos em cirurgias eletivas (médico ortopedista de SPA).

Quando se analisa a articulação da rede pré hospitalar com a rede hospitalar e os seus mecanismos próprios de transferência e transporte, a situação se apresenta até certo ponto adequada ao perfil das unidades. A integração intersetorial, seja com as áreas de ação social, educação, esporte, seja com as de justiça, direitos humanos, segurança e turismo, parece estar em processo de articulação nas unidades do PSF e nos PS 24h, enquanto nos ambulatórios especializados a integração já aparece em maior número, talvez por eles disporem de profissionais mais sensibilizados a essas questões. Do ponto de vista do modelo assistencial vigente, são as equipes do PSF que devem desenvolver prioritariamente essa ação.

Observou-se que tanto o setor saúde quanto o setor extrassetorial (Ministério Público, delegacias, Consel hos de Direitos, Secretaria de Ação Social e organizações não governamentaisONGs) encaminham os pacientes idosos prefe rencialmente para os ambulatórios especializados, sendo estes de fato as unidades de referência a essa população.

Nos casos de violência contra idosos, quatro unidades do PSF, um PS 24h, dois ambulatórios especializados e o Samu afirmaram oferecer suporte e/ou acompanhamento às vítimas; entretanto, nenhum deles avaliou o serviço como adequado ou suficiente. Duas unidades de PSF também realizam 0 atendimento aos vitimizadores. 0 atendimento às famílias dos idosos vítimas de violências é realizado em apenas um ambulatório especializado e três PSF. Esta é uma questão que perpassa a compreensão da violência intrafamiliar e que carece de uma nova abordagem em que a família deve ser o foco de atenção e requer 0 envolvimento de uma equipe de saúde multiprofissional.

A penas o Samu dispõede protocolo clínico de atendimento a idosos vítimas de acidentes e violências. Isso refletea falta de um modelo assistencial vinculado ao padrão epidemiológico da violência em nível local, uma vez que Recifeapresentou em 2006 um coeficiente de mortalidade por causas externas de 173,1 por cem mil habitantes?.
0 gestor do centro de referência afirma quea rede de atenção não está estruturada para atender a população de acordo com cada ciclo de vida e principalmente os idosos vítimas de violência, bem como contemplar medidas de prevenção e promoção para os agravos à pessoa idosa. Contudo, o entrevistado não concorda que se estruture particularmente para o idoso um serviço/ programa voltado para atender vítimas de violência. Penso quenão caberia ao SU S se responsabilizar pelos determinantes da violência no idoso e sim sua inclusão social, e essa ação deveria ser desenvolvida por uma rede intersetorial que pensasse a prevenção da violência e promoção da saúde, o que envolve ações no entorno da moradia, ações voltadas para a família e o seu ambiente social.

Atenção integral e integrada à saúde da pessoa idosa; assistência interdisciplinar e intersetorial às vítimas de acidentes e violência

O Estatuto da Pessoa Idosa ${ }^{16}$ assegura a atenção integral à saúde do idoso, por intermédio do Sistema Ú nico de Saúde (SUS), garantindo-Ihe o acesso universal eigualitário, em conjunto articulado econtínuo das ações eserviços, para prevenção, promoção, proteção e recuperação da saúde.

Para a análise dos serviços de nível hospitalar que atendem idosos em situação de acidentes e violências na cidade do Recife, foram estudadas oito unidades de saúde. Do total de unidades estudadas, seis contam com Unidade de Tratamento Intensivo (UTI) e cinco apresentam Unidade de Tratamento Semi-Intensivo. Como a recomendação da PNRM AV ${ }^{9}$ é de que todas as unidades hospitalares tenham UTI, há o entendimento de que as unidades hospitalares no Recifeestão adequadas. Além disso, todas as seis UTI têm profissional médico para atendimento 24 horas por dia, ocupam área física própria, possuem acesso facilitado e são de alta complexidade.

As oito unidades estudadas possuem 2.975 leitos hospitalares, dos quais cerca de 35\% são leitos de urgência/emergência, 40\% são destinados a adultos e 3,2\% são leitos deUTI. Estesúltimos estão abaixo do recomendado pela Portaria ํo $1.101^{17}$, que estabelece a necessidade de $4 \%$ a $10 \%$ de leitos deUTI do total de leitos hospitalares (média para municípios grandes, regiões etc.).

Dos 581.691 atendimentos real izados no ano de 2006, 41.803 foram em idosos, correspondendo a $7,2 \%$ do total. Destes, $27 \%$ foram decorrentes de acidentes e $10 \%$ motivados por violência. Com a soma dos percentuais de atendimentos 
por acidentes e violências. temos que quase $40 \%$ dos atendimentos a idosos foram decorrentes desses agravos. Os percentuais podem ser considerados elevados, ainda mais se forem considerados os sub-registros existentes e os registros baseados a partir de procedimentos e lesões e não da causa básica.

Quanto a recursos tecnológicos mínimos e indispensáveis, conforme recomen dação da Portaria GM n ${ }^{\circ} 2.048^{18}$, que regulamenta os sistemas estaduais de urgência e emergência, quase todas as unidades possuem o mínimo recomendado. D os oito hospitais estudados, todos contam com equipamento de eletrocardiografia e radiologia convencional. Ademais, aproximadamente $90 \%$ dos hospitais estudados possuem análise clínica/ ambulatorial, ultrassonografia, ecocardiografia, intensificador de imagem e cirurgia geral. Escassos são os serviços de hemodinâmica, cirurgia ginecológica e banco de tecidos.

De acordo com o levantamento de profissionais disponíveis para atendimento, destaca-se a presença detécnico/auxiliar deenfermagem, seguida deenfermeiro, clínico geral ecirurgião geral. Os profissionais encontrados em menor número foram geriatra, hematologista e toxicologista.

Quanto aos mecanismos próprios de transferência e transporte de pacientes, seis unidades não apresentam articulação com outros serviços. Todos os hospitais contam com suporteininterrupto para laboratório de radiologia e patologia clínica.

A penas uma das oito unidades estudadas oferece estrutura adequada para a permanência de acompanhante familiar do idoso, e nenhuma unidade conta com equipe para internação e/ou assistência domiciliar.

Praticamente todas as unidades de saúde re ferem receber e encaminhar pacientes. Q uanto ao destino dos encaminhamentos, tiveram mais destaque os hospitais, as del egacias e outras unidades de saúde, emenos o PSF/PACS, M inistério Público e Conselhos de Direitos. Quando a questão versou sobre de quem se recebe, houve equilíbrio entre as áreas.

As centrais de regulação devem constituir um verdadeiro complexo regulador da assistência, ordenando o fluxo geral de necessidade/resposta, e, para tanto, devem organizar a relação entre os vários serviços, incluindo leitos hospitalares, procedimentos de alta complexidade, exames complementares, internações e atendimentos domiciliares, consultas, assistência social, transporte sanitário não urgente, informações e outros serviços e instituições ${ }^{17}$.
A articulação com a central de regulação é presente em sete das oito unidades estudadas. Quando os pesquisados foram solicitados a atribuir uma nota a al guns itens relativos à estrutura dos serviços e dinâmica de funcionamento, a maior nota atribuída foi para a comunicação com a central de regulação e a nota mais baixa ficou para os fluxos de referência e contrarreferência. A boa articulação apresentada anteriormente entreas unidades einstituições e com a central de regulação não condiz com a baixa nota atribuída à referência e contrarreferência, talvez pelo fato de o serviço existir, mas de forma inadequada ou insuficiente.

Nos casos confirmados de violência contra idosos, o suporte e/ou acompanhamento aos idosos, familiares/cuidadores e vitimizadores é oferecido por sete unidades, porém apenas uma delas considera o serviço adequado e nenhuma refereter protocolos clínicos para atendimento a idosos vítimas de acidentes e violências.

\section{Estruturação econsolidação do atendimento voltado à recuperação eà reabilitação}

Os serviços de reabilitação estudados no Recife foram sete, na sua mai oria classificados como de nível primário, apenas um deles de nível intermediário e nenhum de referência. $\mathrm{Na}$ lógica de estruturação e organização do sistema de saúde, os serviços de nível primário devem existir em maior número na tentativa de garantir 0 acesso à população, a proximidade geográfica e a prevenção de agravos e sequelas.

Entre os serviços, cinco pertencem à gestão municipal, um é vinculado ao governo estadual, e um conveniado ao SUS. A distribuição aponta a consonância da organização dos serviços com o processo de municipalização da saúde, porém a rede conveniada se mostrou aquém do esperado, uma vez que a contribuição dessa rede na assistência em reabilitação é geralmente ampla.

Existeuma demanda de pacientes encaminhada para as unidades de reabilitação pela central de regulação; essa demanda de pacientes é agendada pela rede própria municipal ou pela rede conveniada. Na reabilitação, 0 atendimento agendado prevalece em relação à demanda espontânea, o que não significa a existência de uma oferta organizada. Como afirma o gestor responsável pela reabilitação no Recife: 0 atendimento na nossa redeéum atendimento a uma demanda referenciada pouco dirigida ao idoso... o usuário éaten- 
dido globalmente, não tem uma, um tratamento específico para esse segmento. Pode pegar paciente, um usuário que levou, que sofreu um acidente, vítima de violência; ele é atendido como se fosse uma coisa de rotina do serviço, não tem nenhum, não tem um protocolo espeć́fico, não tem um horário preestabelecido para atender.

Os serviços analisados de nível primário realizam atendimento ambulatorial e não possuem leitos. 0 serviço de nível intermediário possui apenas três leitos, o que denota a precariedade da assistência para os casos mais graves. Essas informações estão divergentes do que consta no Cadastro Nacional de Estabelecimentos de Saúde, segundo o qual Recife possui vinte leitos de reabilitação concentrados em um único hospital de gestão estadual ${ }^{19}$.

A média de atendimentos a idosos nos serviços de reabilitação, em 2006, representou apenas $3,6 \%$ do total de atendimentos, enenhum foi registrado tendo como causa os acidentes ou violência. Esse baixo percentual de registros pode revelar falta de capacitação dos profissionais para realizar o diagnóstico ea ausência de um sistema de informação voltado para o registro das causas dos agravos.

Os serviços de reabilitação física - primeiro nível eintermediário - deverão garantir o acesso aos serviços auxiliares de diagnóstico e terapia, estabelecidos para a Aten ção Básica Ampliada e definidos pela N orma O peracional da Assistência à Saúde do Sistema Ú nico de Saúde (N OASSUS) nº1/2001, compreendendo: patologia clínica, radiologia e ultrassonografia ${ }^{20}$.

No estudo, os tipos de recursos identificados guardam coerência com o querecomendaa N OASSUS 01/200120. Porém, o quantitativo está abaixo do esperado para uma boa cobertura; apenas um serviço possui os recursos necessários para uma unidadedo primeiro nível de referência.

As equipes profissionais compõem-se basicamente de fisioterapeutas e técnicos/auxiliares de enfermagem, e a maioria possui pelo menos um psiquiatra, fonoaudiólogo, psicólogo e terapeuta ocupacional; observou-se carência das demais especialidades.

No que diz respeito à avaliação de órteses, próteses e meios auxiliares de locomoção, apenas três o fazem e também acompanham. Ao que consta, para as próteses ocorrem menos prescrição, treino para uso, dispensação e acompanhamento.

A dispensação de próteses e órteses no município do Recife érealizada por uma insti tuição filatrópica (Associação de Assistência à Criança
D eficiente-AACD) e um hospital estadual. Estão aquém do que preconiza a Portaria do M S/GM no 818: todos os serviços de reabilitação, nos seus diferentes níveis, devem realizar a prescrição, avaliação, adequação, treinamento, acompanhamento e dispensação de órteses, próteses e mei os auxiliares de locomoção ${ }^{21}$.

$\mathrm{Na}$ avaliação de diversos aspectos da assistência, as mai ores notas atribuídas pelos pesquisados foram para insumos básicos emedicamentos. As menores notas foram para órteses, próteses e meios de locomoção, ratificando o quefoi mencionado anteriormente quanto ao número reduzido de unidades que oferecem o serviço.

Apenas uma unidade referiu possuir recursos como mecanismo próprio para transferência e transportes de pacientes, suporte para laboratório de radiologia e patologia clínica. As unidades de saúde estão se articulando com outros serviços para a real ização detais ações, principalmente suporte para laboratório de radiologia.

As atividades realizadas parecem melhor contemplar a estimulação psicomotora, a avaliação e 0 atendimento em fisioterapia e fonoaudiologia. Destacam-se as poucas atividades ligadas ao atendimento em psicologia, nutrição, enfermagem, avaliação funcional, diagnóstico de eletroneuromiografia, potenciais evocados e urodinâmica. Tais informações apontam para o fato de os serviços de reabilitação não contemplarem as necessi dades de saúde da população idosa, e com isso está a não garantia dos princípios da universalidade e integralidade das ações de saúde.

Das sete unidades estudadas, quatro oferecem atendimento voltado à preparação para alta e consideram o atendimento adequado, porém insuficiente. É praticamenteinexistenteo atendimento domiciliar, o acompanhamento pós-alta e o suporte e/ou acompanhamento às equipes que realizam 0 atendimento aos idosos vítimas deacidentes eviolência.

Percebe-seuma dificuldade do serviço em executar atividades fora da sua sede, como também atividades menos ligadas à assistência e suporte global ao paciente. É relatado queno atendimento de reabilitação a equipe geralmente interage com a família do idoso, pois, na maioria dos casos, o paciente é levado ao serviço por algum familiar. Já a participação da família no processo de reabilitação depende de cada caso. Na maioria das vezes, a família corresponde ao que se demanda, porém o fator financeiro tem uma forte influência nessa relação.

0 relacionamento dos serviços de reabilitação com a rede de serviços de proteção social é 
deficiente, o que corrobora a afirmação dos gestores de que no município do Recife ainda não há uma rede de proteção social estruturada.

Capacitação de recursos humanos; formação e educação permanente dos profissionais de saúde do SUS na área de saúde da pessoa idosa

Todos os profissionais que atendem idosos deveriam estar atentos para a possibilidade de maus-tratos, saber como identificar elidar com os casos, além de estar articulados com as redes de proteção, a fim de minimizar o impacto na população idosa22.

Os gestores entrevistados enfatizam a necessidade de melhorias no atendimento aos idosos vítimas deacidentes eviolência prestado no Recife, e sugerem a capacitação dos profissionais e a estruturação para uma articulação mais eficaz da rede de proteção. Como evidencia o seguinte depoimento: 0 serviço público voltado para 0 atendimento do idoso é avaliado como precário pelas condições de trabalho, e pela falta de corpo médico e de enfermagem com mais atenção aos idosos nos hospitais durante 0 internamento, devido às suas comorbidades.

A pesar da indicação da necessidade de capacitação para identificação e atendimento a casos de idosos vítimas de acidentes e violência, e de as diretrizes das políticas anteriormente mencionadas contemplarem essas ações, são poucas as iniciativas ou avanços nessa perspectiva. Por sua vez, merecem destaque o curso a distância sobre Impactos da Violência na Saúde, promovido pelo Centro Latino-Americano deEstudos deViolência e Saúde Jorge Careli (Claves/Ensp/FiocruzRJ), e a estruturação de grupos e linhas de pesquisa em programas stricto sensu em algumas universidades do país.

Ao setor de saúdenão cabe unicamentea responsabilidade de lutar contra a violência; entre tanto, eledeveexercer um papel importante, tanto na promoção, prevenção eatendimento quanto na capacitação de seus profissionais para o enfrentamento do problema, respaldados na compreensão das relações sociais conflituosas, principalmenteintrafamiliares ${ }^{23}$.

No estudo, a maioria das unidades não notifica casos suspeitos ou confirmados de violência contra idosos. 0 despreparo do profissional em lidar com as vítimas que recorrem ao seu serviço sedeve, possivelmente, ao desconhecimento acerca de como proceder à frente desses casos, como também por não ter para ondeencaminhar. Sabe- se que a dinâmica familiar, como espaço para a geração da violência contra o idoso, e os processos macrossociais e estruturais, como a falta de emprego, a pobreza, a falta de escolaridade, a ausência de albergues para idosos, entre outros, são processos sociais que requerem investimentos em longo prazo e definição política para o investimento em políticas sociais. Contudo, os mecanismos de notificação são importantes barreiras para a identificação, e o registro dos casos de abuso e negligência contra idosos irá contribuir entre outros meios de intervenção para a prevenção e o tratamento de casos que estão a exigir al guma ação ${ }^{24}$.

A notificação éum poderoso instrumento de política pública, uma vez que ajuda a dimensionar a questão da violência em família, a determinar a necessidade de investimentos em núcleos de vigilância e assi stência e ainda permite o conhecimento da dinâmica da violência domésti$\mathrm{ca}^{25}$. Contudo, é fato que a conscientização da sua importância, a quebra de ideias preconcebidas e o treinamento correto para diagnosticar situações de violência são condições necessárias para que o profissional de saúde seja capaz de detectar e notificar, a quem for competente, essa realidade que se apresenta de forma tão expressiva no cotidiano dos seus atendimentos, seja qual for a sua área de atuação ${ }^{26}$. Como confirma a fala do gestor de saúde do município: A rede de saúde está se estruturando para notificar. Contudo, o enfrentamento da violência contra o idoso passa por questões que abrangem a Secretaria de Política Pública, Serviço Social, Segurança, Direitos Humanos etc. Para se estabelecer ações resolutivas com a prática das notificações, é necessário uma rede intersetorial e não atuações isoladas entre os órgãos competentes.

De acordo com o Estatuto da Pessoa Idosa ${ }^{16}$, a prevenção e a manutenção da saúde do idoso serão efetivadas por meio de, entre outros, atendimento geriátrico e gerontológico em ambulatórios e unidades geriátricas de referência, com pessoal especializado nas áreas de geriatria e gerontologia social. Do total de unidades estudadas, menos da metade apresentam profissionais com formação em gerontologia/geriatria, o que remete ao déficit na formação de geriatras no Brasil, com poucos programas de residência.

Praticamente todos os profissionais que representaram as unidades já ouviram falar da Política Nacional de Saúde da Pessoa Idosa e do Estatuto do Idoso, porém a leitura e o aprofundamento nos documentos são tarefas realizadas por menos da metade dos profissionais. Acres- 
centa-se que a PNRM AV foi o documento me nos conhecido elido.

\section{Consideraçõesfinais}

As duas políticas usadas como principal referência para 0 estudo abordam a necessidade de organização, reestruturação e ampliação da rede de assistência em saúde à pessoa idosa e vítima de acidentes e violência, em todos os níveis de atenção, a fim de contribuir com o cumprimento da integralidade da assistência. No entanto, a rede assistencial no Recife, incluindo atenção pré hospitalar, hospitalar e de reabilitação, não está organizada de forma a assistir, especificamente, a pessoa idosa vítima de acidentes e violência.

Observou-se que as diretrizes das políticas estudadas são contempladas apenas parcialmente; há que se avançar na organização de estrutura, número de leitos, transporte e transferência de pacientes, referência e contrarreferência, central de regulação, diversidade profissional, profissionais com formação em geriatria, capacitação profissional e cobertura em reabilitação.

Embora o número de atendimento a idosos vítimas de acidentes e violência tenha sido el eva- do, observou-se a inadequação no atendimento com relação aos seguintes itens: deficiência de estrutura adequada para a permanência do acompanhante do idoso; ausência de protocolos clínicos específicos para o atendimento nesses casose de fichas de notificação; inadequação do suporte aos idosos, cuidadores e vitimizadores; pouco envolvimento dos ambulatórios especializados; capacitação profissional insuficiente e inexistência de um fluxo definido para tal população.

Destaca-se a necessidade de profissionais e gestores sensibilizados para a abordagem adequada da violência contra idosos, em quea família deve ser o foco de aten ção em uma assistência integral, intersetorial einterdisciplinar. A melhoria da atenção à pessoa idosa vítima de acidentes e violências será um passo importante para o cumprimento das políticas públicas de saúde, e com isso um caminho a ser trilhado para além do campo das invisibilidades.

Essa anál ise pode representar um instrumento passível de contribuir na reordenação do sistema local de saúde, reconhecendo a pessoa idosa como vulnerável a acidentes e violência. Assim, o idoso passa a ser percebido em suas especificidades, particularidades, fragilidades e fortalezas, contem plado em seu direito à cidadania e a uma vida digna. 


\section{Referências}

1. Instituto Brasileiro de Geografia e Estatística. Censo Demográfico 2000. [site na internet]. [acessado 2009 jun]. Disponível em: http://www.ibge.gov.br

2. Organización Mundial de la Salud. Informe sobre la salud en el mundo. Ginebra: OMS; 2002.

3. Debert GG. A reinvenção da velhice: socialização e processos de reprivatização do envelhecimento. São Paulo: Editora da Universidade de São Paulo, 2004.

4. Gawryszewski VP, Jorge MHPM, Koizumi MS. $M$ ortes e internações por causas externas entre os idosos no Brasil: o desafio de integrar a saúde coletiva e atenção individual. Rev Assoc M ed Bras 2004; 50(1):97-103.

5. Katz M, Okum AMAA, Santos ALG, Guglielmetti CLB, Sakaki MH, Zumiotti AV. Epidemiologia das lesões traumáticas de alta energia em idosos. Acta Ortop Bras 2008; 16(5):279-283.

6. Brasil. Presidência da República. Subsecretaria de Direitos Humanos. Plano de Ação para o Enfrentamento da Violência Contra a Pessoa Idosa. Brasília: Subsecretaria de Direitos Humanos; 2005.

7. M inayo MCS. Violência contra idosos: relevância para um velho problema Cad Saude Publica 2003; 19(3):783-791.

8. Brasil. Departamento de Informática do SUS. Indicadores de morbidade e mortalidade. Recife, PE, 20052006. [site na internet] [acessado 2008 abr]. Disponível em: www.datasus.gov.br

9. Brasil. Ministério da Saúde. Política Nacional de Redução da M orbimortalidade por Acidentes e Violências. Portaria GM/M S no 737, de 16 de maio de 2001. Diário Oficial da União 2001; maio 18.

10. Brasil. Ministério da Saúde. Portaria MS/GM n 2.528 , de 18 de outubro de 2006. Política Nacional de Saúde da Pessoa Idosa. Diário Oficial da União 2006; out. 20.

11. Denzin NK. The research act. Chicago: Aldine; 1973.

12. Minayo MCS, Assis SG, Souza ER, organizadores. Avaliação por triangulação de métodos: abordagem de programas sociais. Rio de Janeiro: Editora Fiocruz; 2005.

13. Bardin L. Análise de conteúdo. Lisboa: Edições 70; 1979.

14. M inayo MCS. 0 desafio do conhecimento: pesquisa qualitativa em saúde. 8a ed. São Paulo: Hucitec; Rio de Janeiro: Abrasco; 2004

15. Kennedy, RD. Elder abuse and neglect: the experience, knowledge, and attitudes of primary care physicians. Fam M ed 2005; 37(7):481-485.
16. Brasil. M inistério da Justiça. Secretaria Especial de Direitos Humanos. Estatuto da Pessoa Idosa. Brasília: Secretaria Especial de Direitos Humanos; 2003.

17. Brasil. M inistério da Saúde. Portaria GM no 1.101 , em 12 de junho de 2002. Diário Oficial da União 2002; 13 jun.

18. Brasil. M inistério da Saúde. Portaria GM n².048, 5 de novembro de 2002. Política Nacional de Atenção às U rgências. Braślia: M inistério da Saúde; 2004.

19. Brasil. M inistério da Saúde. Cadastro Nacional de Estabelecimentos de Saúde. [site na Internet]. [acessado 2009 jun 20]. Disponível em: www.cnes. datasus.gov.br

20. Brasil. Ministério da Saúde. Portaria M S/GM no 95, de 26 de janeiro de 2001. Norma Operacional da Assistência à Saúde/NO AS-SUS nº 01/2001. Brasília: M inistério da Saúde; 2001.

21. Brasil. M inistério da Saúde. Portaria MS/GM nº 818, de 5 de junho de 2001. Organização e implantação de Redes Estaduais de Assistência à Pessoa Portadora de Deficiência Física. Brasília: Ministério da Saúde; 2001.

22. M cAlpine $\mathrm{CH}$. Elder abuse and neglect. Age and Ageing 2008; 37:132-133.

23. Leal SM C, Lopes MJM. A violência como objeto da assistência em um hospital de trauma: o "olhar" da enfermagem. Cien Saude Colet 2005; 10(2):419-431.

24. Taylor DK, Bachuwa G, Evans J, Jackson-Johnson $V$. Assessing barriers to the identification of elder abuse and neglect: a communitywide survey of primary care physicians. Journal of the $\mathrm{N}$ ational $\mathrm{M}$ edical Association 2006; 98(3):403-404.

25. Gonçalves HS, Ferreira AL. A notificação da violência intrafamiliar contra crianças e adolescentes por profissionais da saúde. Cad Saude Publica 2002;18(1):315-319.

26. Saliba O, Garbin CAS, Garbin AJI, Dossi AP. Responsabilidade do profissional de saúde sobre a notificação de casos de violência doméstica. Rev Saude Publica 2007; 41(3):472-477.

Artigo apresentado em 09/07/2009

Aprovado em 28/08/2009

Versão final apresentada em 14/10/2009 